RECEIVED BY TIC SEP 241979

GA-A15518

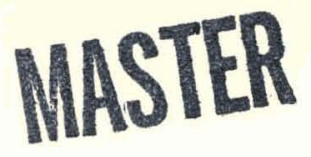

\title{
LIFE-LIMITING ASPECTS OF GCFR CORE ASSEMBLY DESIGNS
}

\author{
by \\ M. P. LaBAR
}

JULY 1979 


\section{DISCLAIMER}

This report was prepared as an account of work sponsored by an agency of the United States Government. Neither the United States Government nor any agency Thereof, nor any of their employees, makes any warranty, express or implied, or assumes any legal liability or responsibility for the accuracy, completeness, or usefulness of any information, apparatus, product, or process disclosed, or represents that its use would not infringe privately owned rights. Reference herein to any specific commercial product, process, or service by trade name, trademark, manufacturer, or otherwise does not necessarily constitute or imply its endorsement, recommendation, or favoring by the United States Government or any agency thereof. The views and opinions of authors expressed herein do not necessarily state or reflect those of the United States Government or any agency thereof. 


\section{DISCLAIMER}

Portions of this document may be illegible in electronic image products. Images are produced from the best available original document. 


\section{NOTICE}

This report was prepared as an account of work sponsored by the United States Government. Neither the United States nor the Department of Energy, nor any of their employees, nor any of their contractors, subcontractors, or their employees, makes any warranty, express or implied, or assumes any legal liability or responsibility for the accuracy, completeness or usefulness of any information, apparatus, product or process disclosed, or represents that its use would not infringe privately owned rights. 


\title{
GA-A15518
}

\section{LIFE-LIMITING ASPECTS OF GCFR CORE ASSEMBLY DESIGNS}

\author{
by \\ M. P. LaBAR
}

This is a preprint of a paper presented at the Helium Breeder Associates/Department of Energy GCFR Program Technical Review Meeting, May 31, 1979, Rancho Bernardo, California, and to be published in the Proceedings.

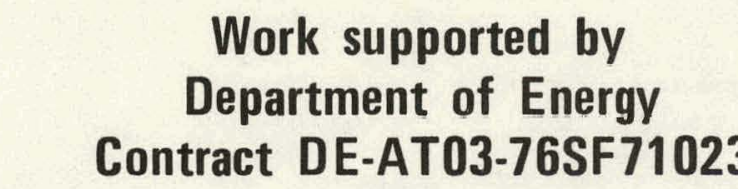

GENERAL ATOMIC PROJECT 6113

JULY 1979

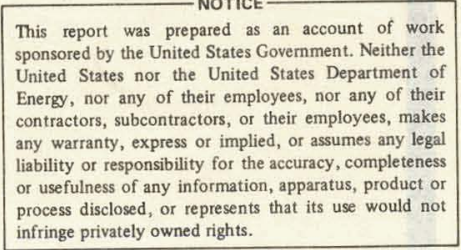

infringe privately owned rights. 
LIFE-LIMITING ASPECTS OF GCFR CORE ASSEMBLY DESIGNS*

M. P. LaBar

General Atomic Company

San Diego, California

ABSTRACT

A review is made of the various potential life-limiting effects of current consideration in the GCFR core assemblies. The most life-limiting aspects are not related to individual fuel rod behavior characteristics, but rather to distortions within the rod bundles. Fuel rod internal fission gas is not limiting, as it is in most fast reactors, because of the GCFR pressure-equalization system. The life-limiting bundle distortions are the result of neutron-flux-enhanced creep and fast-fluence-induced metal swelling. These effects are somewhat unique to fast reactors and are material dependent. The current evaluations apply to the use of $20 \%$ cold-worked type 316 stainless steel as the core structural material. Alternate structural materials having lower neutron-fluence-induced metal. swelling would reduce the distortions, in which case, the most life-limiting effect would likely be one of the more traditional effects such as fuelclad mechanical interaction (FCMI).

INTRODUCTION

The principal life-limiting considerations of nuclear reactor fuels in the form of cylindrical metal-clad fuel rods are generally associated with operational effects which apply to individual rods. These operational effects include clad collapse, buildup of internal fission gas pressure, and fuel-clad mechanical interaction (FCMI). For the liquid metal fast breeder reactor (LMFBR), the bulk of the fuel lifetime research and development effort has been focused upon effects associated with fission gas pressure buildup. The rod internal fission gas pressure has

${ }^{\star}$ Work supported by the Department of Energy, Contract DE-AT03-76SF71023. 
been considered the most limiting lifetime characteristic in the LMFBR primarily because the coolant pressure is low, the burnup is high, and there is almost $100 \%$ release of the gaseous fission products.

The fission gas pressure lifetime limitation does not exist in the design of the gas-cooled fast breeder reactor (GCFR). The helium coolant system used in the GCFR permits the use of a pressure equalization system (PES) which equalizes the pressure within the fuel rods to the external coolant pressure. Consequently, the primary life-limiting consideration of LMFBR fuels is eliminated in the GCFR.

The question is, therefore, what are the life limitations of the GCFR core assemblies? The purpose of this paper is to describe those lifelimiting considerations of current concern in the GCFR. Many of the lifelimiting aspects are not related to individual rod behavior but rather to interaction within or between bundle assemblies. These effects are somewhat unique to fast reactors and are, in general, the consequence of neutron-flux-enhanced creep and fast-fluence-induced metal swelling.

For presentation of the life-limiting effects of current concern in the GCFR, the fuel rod performance characteristics are first reviewed; intraassembly followed by interassembly interaction considerations are covered; and finally, the potential use of alternate structural materials is briefly described. It is anticipated that this information will be useful in provlding some guidance for fucure GCFR research and development efforts.

FUEL ROD CONSIDERATIONS

\section{Traditional Effects}

The principal traditional life-limiting consideration in fast reactor fuel rods is buildup of internal fission gas pressure. Another potential life-limiting mechanism which has generally received secondary attention is FCMT. In the GCFR, the buildup of internal fission gas preseurc is 
eliminated by the PES (Ref. 1). In the PES, the fuel rods are vented in a fashion that results in equilibrating the rod internal pressure to the core exit pressure.

Compared to an LMFBR fuel rod, the fuel in a GCFR rod is subjected to a high static pressure (the coolant system pressure of approximately 85 atm) and there is only a small ( $<3 \mathrm{~atm})$ pressure differential across the clad wall. To evaluate the effect of the differing pressure conditions in a composite fuel rod, two irradiation behavior analyses were performed of breeder reactor fuel rods clad with $20 \%$ cold-worked (CW) 316 stainless steel using the LIFE-3 fuel rod performance code (Ref. 2). The first analysis represented the GCFR vented fuel rod operating conditions. The second analysis was identical to the first, except that the fuel rod internal pressure was allowed to build up in a fashion representative of the LMFBR sealed rod behavior; the primary coolant system pressure was also set equal to a value representative of the LMFBR.

The pellet-to-clad gap results are plotted as a function of burnup in Fig. 1. In the sealed rod design, the initial open gap decreases quite rapidly to zero and remains closed until a burnup of approximately 3.5 at \% is reached. During this period of gap closure, FCMI occurs. After 3.5 at \% burnup, the gap reopens and continues to enlarge to the end of life. In the vented rod, somewhat different gap behavior is obtained. The initial open gap tends to close, like that in the sealed rod; however, it never closes cumpletely because the high static gas pressure tends to restrain the gaseous fission product swelling. The vented rod gap begins to enlarge at about the same burnup as the gap opened up in the sealed rod, but at lower rates. The results indicate that the vented rod has less potential for FCMI and the rod expanstons are less due to the absence of internal pressure buildup at end of life.

To confirm the results predicted by the LIFE performance models, irradiation test data are needed on fuel swelling characteristics as a function of static pressure. Argonne National Laboratory (ANL) has resently proposed a sericc of EBR II irradiations (Ref. 3) whlch can 
possibly be used to obtain the required data. Although the behavior predicted by LIFE-3 in Fig. 1 requires further experimental verification, the qualitative trend can be easily explained by fuel swelling theories.

Cesium Accumulations

Test irradiations of fast reactor fuel rods have shown that there is a tendency for the formulation of low-density Cs-U-O compounds at local sites within the rods. Theoretical evaluations as well as test irradiations indicate there is the potential for fission product cesium to migrate to lie fuel-blanker interface and to react locally with the blanket urania (Ref. 4).

There are at least two concerns associated with local cesium compound accumulations. These are (1) that volume expansions resulting from the lower density compounds can cause excessive clad strain, and (2) that axial gas-transport paths within the fuel rod can become blocked. A gas flow restriction was, in fact, observed in the GCFR test irradiation GB-10 sweep gas experiment (Ref. 5). The gas restriction has been traced to a blockage at the fuel-blanket interface. Local clad strains have also been observed in test irradiations in the region of the fuel-blanket interface (Ref. 6).

The effect of local cesium accumulations within fuel rods is considered to be a generic problem for all fast oxide reactors. Occasionally, it is addressed as being a particular problem for the GCFR since a gas venting system is used to prevent the buildup of pressure within. the rods. However, the fission gas within an LMFBR rod must also communicate with an end plenum to avoid overstraining the cladding.

Local cesium accumulations are not expected to result in life limitations. We anticipate that means can be developed to avoid or accommodate the accumulations. For example, in the GCFR F-5 test irradiation, presently in progress, special interface blanket pellets are included which 
are of a low-density design to provide voidage to accommodate cesium compounds. Other possibilities include adjustments in the oxygen/metal ratio and smear density in the fuel. In the GB-10 experiment, where a gas flow' restriction was found, it was also noted that the gas flow conductance was restored with each power variation. Consequently, if formulation of cesium compounds cannot be avoided, it can probably be accommodated by design or operational provisions, without significant penalty. Further development efforts are, however, required.

INTERASSEMBLY CORE DISTORTIONS

There are basically two forms of core distortions that can result in life limitations: the first is duct dilation, and the second is assembly bow.

Duct Dilation

Duct dilation is the expansion of the hexagonal flow duct due to operational effects, as shown in Fig. 2. The two basic components of duct dilation are uniform duct expansion and bulging of the hexagonal flats. The generally uniform duct dilations are caused by thermal expansion and metal swelling induced by fast fluence. The bulging component of dilation is caused by the internal pressure-induced stresses in the presence of neutron-flux-enhanced creep.

The primary life-limiting aspect associated with duct dilation is that if the ducts dilate too much, interactions will occur between adjacent assemblies to the extent that they will bind with each other during refueling. To avoid binding, the criterion has been selected for the GCFR that the interassembly gap and dur.t wall. thickness be sized to prevent diametrically opposed duct contact at the most restrictive location (e.g., at the restraint pads) during assembly removal. Contact on only one side is permitted, and credit can be taken for variation in adjacent assembly lifetimes. The reference fuel management scheme results in replacing every 
third assembly in the active core every year. As a result there are no two adjacent assemblies of the same lifetime.

Figure 3 is a plot of various combinations of interassembly gap and duct wall thicknesses that satisfy the above criterion for a downflow GCFR core. The results show that a reasonable duct wall thickness and interassembly gap can be chosen for the given design lifetime. A 4-mm duct wall thickness and a 10-mm interassembly gap have tentatively been selected. Similar analyses have not yet been performed for the upflow core; however, Fig. 3 illustrates that appropriate parameters can be selected such that duct dilation is not life limiting.

Assemb1y Bow

A general illustration of assembly bow for the GCFR upflow core configuration is shown in Fig. 4, which has been taken from Ref. 7. These distortions are caused by radial thermal gradients and differential fluenceinduced metal swelling. The tentative design criteria selected for evaluation of the GCFR upflow core distortions are as follows:

1. Assembly interaction loads at refueling (i.e., shutdown conditions) $\leq 2200 \mathrm{~N}$.

2. Assembly total distortion (for control rod insertion and drive interfacing) within an envelope $10 \mathrm{~mm}$ larger on each side of assembly nominal size.

3. Assembly interaction at full power on at least one load pad throughout life.

4. Minimal positive reactivity interaction.

Scoping studies of the GCFR upflow configuration by means of the NUBOW 2-D computer program (Ref. 8) have shown that the above criteria can be sat1sfled using a nominal beginning-of-life cold interassembly clearance of $0.8 \mathrm{~mm}$ at the load pads (Ref. 9). Considerably more effort is required to confirm these initial results. However, present indications are that designs can be established that will satisfy selected lifetime requiremente with regard to asscmbly bow corc distortions. 
INTRAASSEMBLY DEFORMATIONS

There are several intraassembly operational effects that are of current concern with regard to their potential life-limiting aspects. They include rod bowing; rod-spacer friction and wear, differential expansion, and fretting; and skcleton structure creep ratcheting. All of these are some form of, or consequence of, interaction between components, principally the fuel rods and the fuel rod spacer grids. These effects are sometimes referred to as composite bundle effects or, simply, bundle effects. These bundle effects are discussed in following sections.

$\underline{\text { Rod Bowing }}$

The edge rods on the perimeter of the rod bundle adjacent to the flow duct wall are subjected to transverse thermal gradients due to the different coolant channels on the duct side and bundle side of the rods. As a result of the thermal gradients, thermal bow occurs, and, because fluence-induced metal swelling is temperature dependent, a metal swelling bow eventually develops.

A number of studies have been performed to evaluate edge rod bowing in the GCFR fuel assemblies. The following are the primary criteria that have been used in evaluation of the results:

1. Rod-to-rod gap $\geq 1 \mathrm{~mm}$ and rod-to-duct gap $\geq 0.5 \mathrm{~mm}$ (Ref. 10). This criterton has been selected from the results given in Fig. 5.

2. Rod-spacer interaction force $\leq 9 \mathrm{~N}$.

The results of a recent analysis are given in Fig. 6. Two different analyses are shown, with and without duct dilation. The results without duct dilation satisfy the applicable criteria throughout life. However, the gap clearance criteria are not satisfied when the effects of duct dilation are included in the analysis. To date, no satisfactory solution has been found for satisfying rod bow for the design lifetime of $100 \mathrm{MWd} / \mathrm{kg}$ 
using the reference $20 \%$ CW 316 stainless steel structural material for the cladding. Rod bow appears to be limiting lifetime to approximately $75 \mathrm{MWd} / \mathrm{kg}$.

\section{Rod-Spacer Friction and Wear}

During operations which involve change of power, temperature levels within the core assemblies change. Differential temperature changes between the fuel rods and structural supports cause axial motions of the rods through the spacer grids. Since the rod surfaces are roughened to enhance heat transfer, rod-spacer interaction tests involving lifetime axial motions were initiated some time ago. In the tests run to date, the transverse force due to. rod bowing has been simulated. Friction and wear data have been gathered which indicate acceptable behavior throughout lifetime.

The effect of rod-to-spacer misalignment angle has not yet been experimentally investigated. Rod bow is one effect that contributes to the misalignment angle; other contributors are the result of fabrication tolerances. A data base on rod-spacer friction, including the effects of misalignments, is needed for assessing the lifetime characteristics of the spacer grids and grid support systems.

\section{Rod-Spacer Differential Expansion}

During operation, the fuel rod cladding temperatures are somewhat hotter than the temperatures of the grids that are used to maintain the rod lateral spacing. The fluence-induced metal swelling is temperature dependent and, in the region of most importance, swelling increases with increasing lemperature. Consequently, differential swelling between fuel rods and spacer grids is predicted. Figure 7 contains representative results for a nominal end-of-life case where no account has been taken of the local temperature perturbations caused by the presence of the grids. The results indicate that excessive clearance is required to achieve full design lifetime for the present reference spacer grid design. 
Design alternatives and performance analyses need further effort to avoid this potential life-limiting effect with the present reference structural material.

$\underline{\text { Rod-Spacer Fretting }}$

To accommodate rod-to-spacer misalignment effects, such as rod bow, and rod-spacer differential expansions, the clearance between the rod and spacer grid contact pads needs to be on the order of at least $0.25 \mathrm{~mm}$. At present, inadequate experimental data are available on the flow-induced vibration characteristics of rods in parallel gas flow (Ref. 11); the presence of small gaps between the rods and the rod lateral supports is a source of further uncertainty. Consequently, flow-induced vibration tests are needed, and plans have recently been initiated to prepare a helium flow test rig to perform the required tests. Fretting between the rods and rod support points is one aspect of flow-induced vibration that is fundamental for lifetime considerations. Fretting tests consequently will be carried out in parallel with the flow-induced vibration tests.

\section{Skeleton Structure Creep Ratcheting}

As the transverse rod thermal gradients develop in going up to power, interaction forces between the rods and spacer grids develop. The transverse force in the presence of rod axial thermal elongation and friction results in axial as well as transverse loading of the spacer grids. The axial forces are also imposed upon the spacer structural support tie-rods. How long the frictional load will be maintained once full power has been reached is questionable and subject to uncertain effects such as vibration and power fluctuations. The total axial flexibility of the tie-rods, spacer grids, and fuel rods is expected to play a major role in the performance of the system.

If the axial loads developed by friction between the rods and grids in going to power are sustained, flux-enhanced creep can result in stress relaxations and permanent deformations. Subsequent shutdowns and startups 
would be expected to repeat the process. The result would be creep ratcheting of the loaded components, mainly the spacer grids and tie-rods. Depending on the degree of deformation per cycle, creep ratcheting could result in lifetime limitations. Additional development efforts are required before this potential lifetime limitation effect can be adequately quantified.

\section{ALTERNATE MATERIALS}

The most lifetime-limiting effects in the GCFR core assemblies are primarily a consequence of neutron-fluence-induced metal swelling and, to a lesser degree, neutron-flux-enhanced creep. The neutron-fluence-induced metal swelling of the reference $20 \%$ CW 316 stainless steel undergoes an incubation period; essentially no swelling occurs until approximately a fluence of 5 to $9 \times 10^{22} \mathrm{n} / \mathrm{cm}^{2}$ is reached. Consequently, a substantial lifetime, on the order of $50 \mathrm{MWd} / \mathrm{kg}$, can be attained before significant metal swelling occurs. Metal swelling effects for fluences $>1 \times 10^{23} \mathrm{n} / \mathrm{cm}^{2}$ appear to limit achievable GCFR fuel lifetimes to approximately $75 \mathrm{MWd} / \mathrm{kg}$.

Alternate materials are currently under development which appear to have much more favorable performance characteristics (Ref. 12). The primary performance attribute which tends to characterize the alternate materials is lower neutron-fluence-induced metal swelling. Other properties of importance also appear to be satisfactory for the prime alternate material candidates. Present indications are that the GCFR goal burnup of $100 \mathrm{MWd} / \mathrm{kg}$, or greater, is achievable by means of alternate structural materials.

\section{SUMMARY}

There are many different operational effects that could result in lifetime limitation of the GCFR core assemblies. Those of current concern include interassembly distortions (duct dilation and assembly bow) as well as intraassembly deformations (rod-spacer differential expansions and rod bow). Individual GCFR fuel rod lifetimes, in the absence of assembly 
deformation effects, could be limited by FCMI and local cesium accumulations. Other lifetime-limiting considerations include rod fretting and creep ratcheting of the rod bundle skeleton structure.

Present evaluations indicate that the interassembly distortions can be accommodated by suitable cholce of duct wall thickness and interassembly gap, FCMI is less severe than in equivalent LMFBRs, and local cesium concentration effects can probably be accommodated. There are not yet sufficient data to fully evaluate the rod fretting and rod-spacer interaction affects, nor has sufficient analyeis been performed of the skeleton structure creep ratcheting. However, present indications are that these will not be limiting. For $20 \% \mathrm{CW} 316$ stainless steel, the intraassembly deformations, differential rod-spacer expansion and rod bow, appear most limiting, with assembly lifetimes of approximately $75 \mathrm{MWd} / \mathrm{kg}$ as compared to the design goal of $100 \mathrm{MWd} / \mathrm{kg}$. An alternate structural material having less fluence-induced metal swelling could remove this limitation. For alternate materials having lower swelling characteristics, FCMI and/or in-pile creep deformation are expected to become the more lifetime-limiting effects.

\section{REFERENCES}

1. Campana, R. J., "Pressure Equalization System for Gas-Cooled Fast Breeder Reactor Fuel Elements," Nuclear Technology 12; Oct. 1971.

2. "LIFE-III Fuel-Element Performance Code," Energy Research and Development Administration Report ERDA 77-56, July 15, 1977.

3. "GRIST-2 Test Program Plan" (partial draft), Argonne National Laboratory Report S6100-0001-AP, March 1979.

4. "Reactor Development Program Progress Report," DOE Report ANL-RDP-79, Argonne National Laboratory, January 1979, p. 6.1.

5. Longest, A. W., et al., "Results from Irradiation of Vented GCFR Fuel Rods in the GB-9 and GB-10 Capsule Experiments," DOE Report ORNL-5258, Oak Ridge National Laboratory, September 1978. 
6. Karnesky, R. A., "Cesium Migration in LMFBR fuel Pins," Presented at International Conference on Fast Breeder Reactor Fuel Performance, Monterey, California, ISBN: 0-89448-105-3, March 1979.

7. Thomson, J. D., "National Core Restraint Development Program, Westinghouse Core Restraint Test Facility (WCRTF)," ERDA Report WARD-CR-3045-18, Westinghouse Advanced Reactors Division, June 1979.

8. Cha, B. K., et al., "NUBOW-2D Inelastic: A Fortran Program for Static Two-Dimensional Structural Analysis of Bowed Reactor Cores, Including Effects of Irradiation Creep and Swelling," ERDA Report ANL-CT-77-34, Argonne. National Laboratory, June 1977.

9. Lee, G. E., "GCFR Upflow Core Design," Proceedings, GCFR Program Technical Review Meeting, Rancho Bernardo, California, May 1979.

10. "Gas-Cooled Fast Breeder Reactor Quarterly Progress Report for the Period May 1, 1978 through July 31, 1978," DOE Report GA-A15054, General Atomic Company, August 1978, p. 2-32.

11. Henry, C. D., et al., "Flow-Induced Vibrations in GCFR Core Components," Presented at 3rd National Congress on Pressure Vessels and Piping Technology, San Francisco, California, June 1979.

12. "Alloy Property Data Book," HEDL-TC-293, Rev. 3., Hanford Engineering and Development Laboratory, October 1978. 


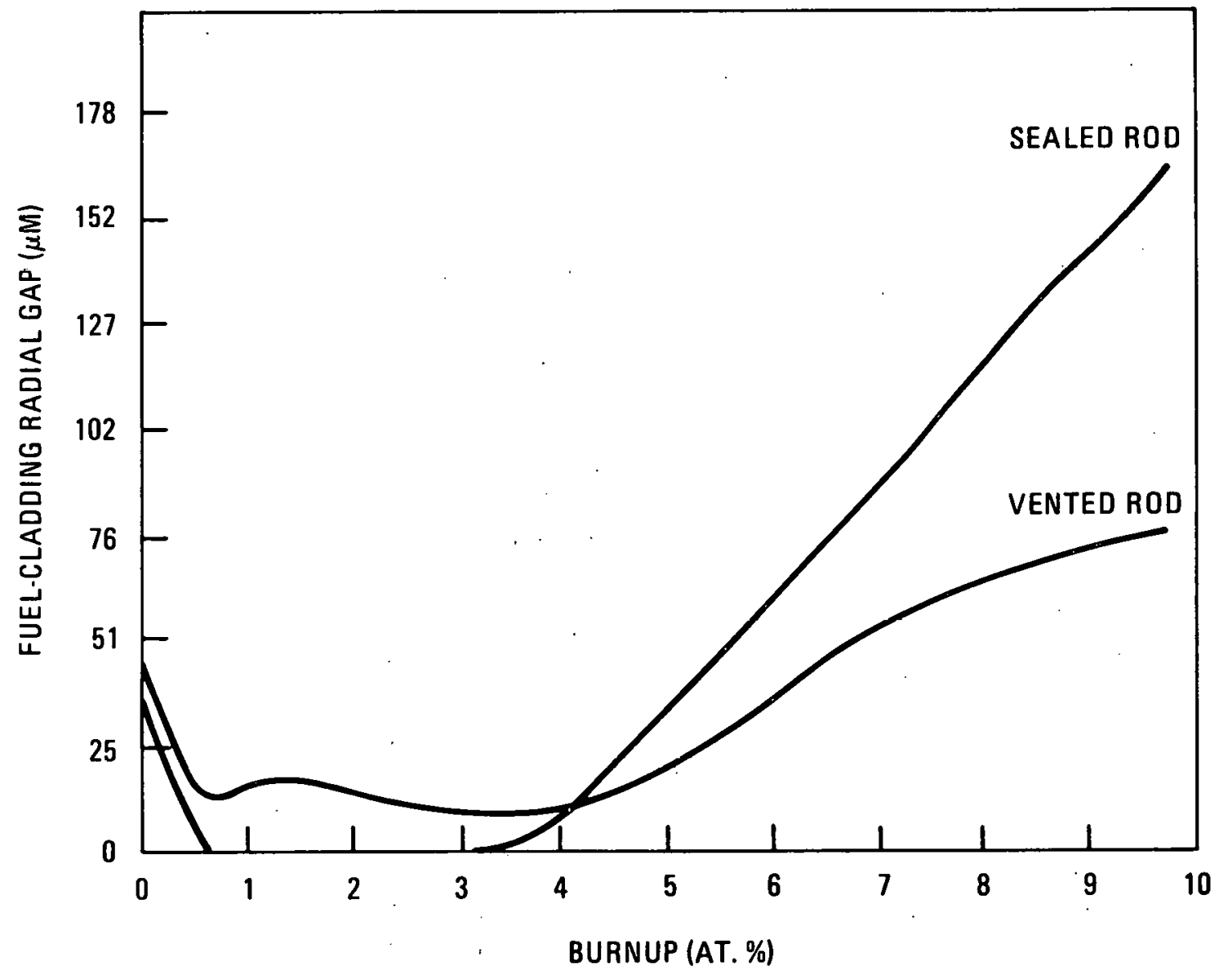

Fig. 1. Steady-state fuel-clad radial gap 


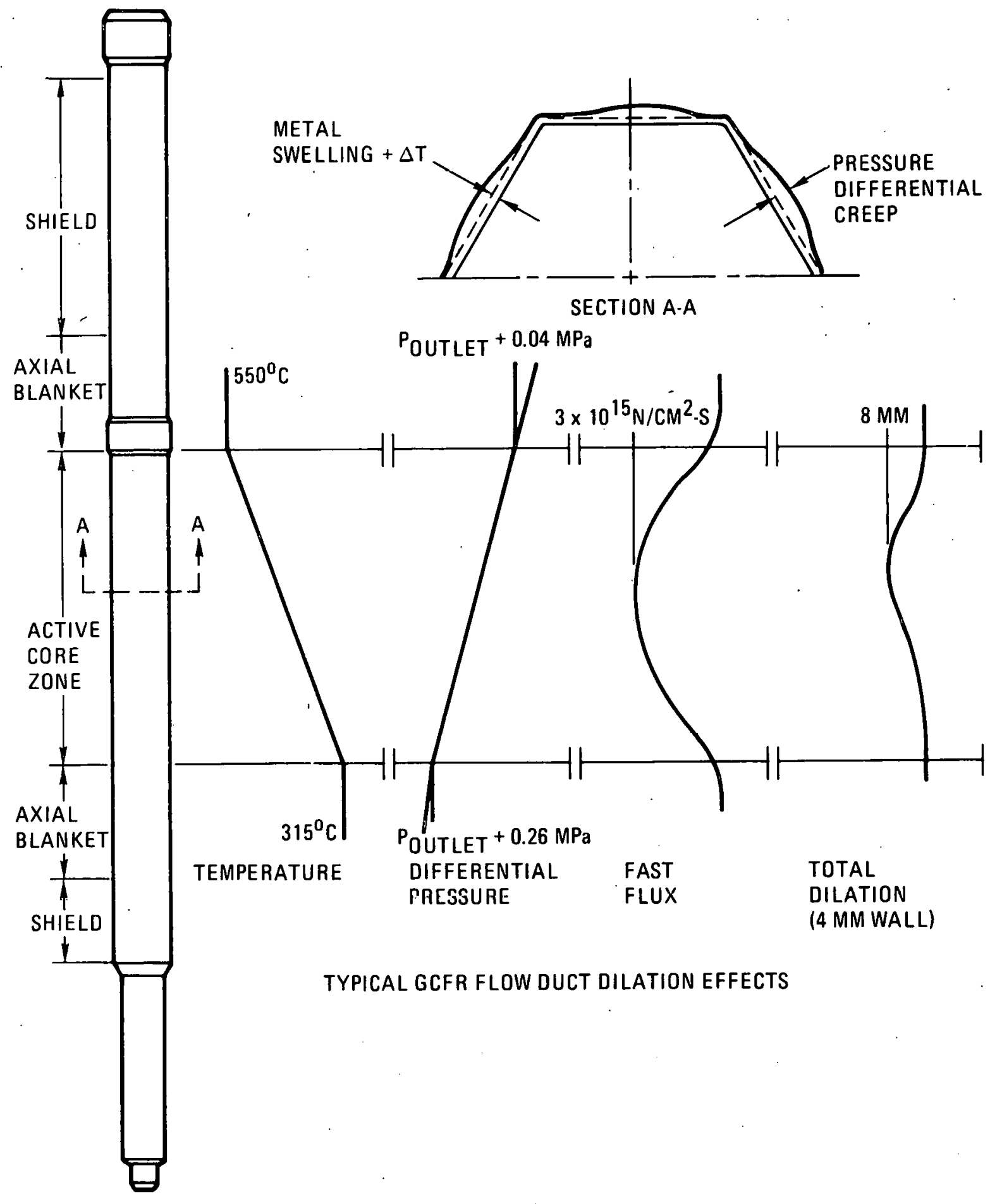

Fig. 2. Flow duct dilation 


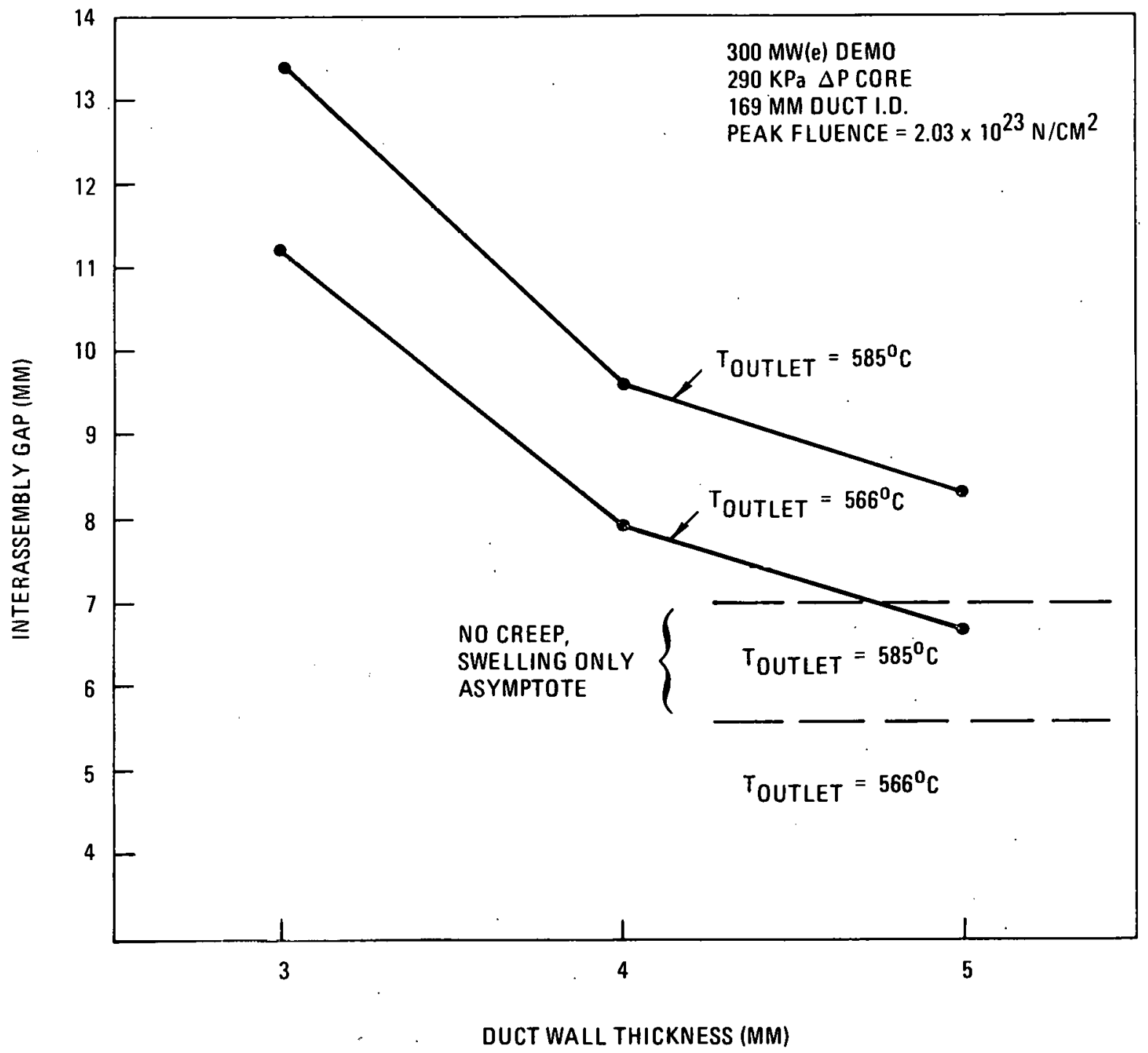

Fig. 3. BOL interassembly gap required to prevent duct-to-duct contact. for various duct wall thicknesses 


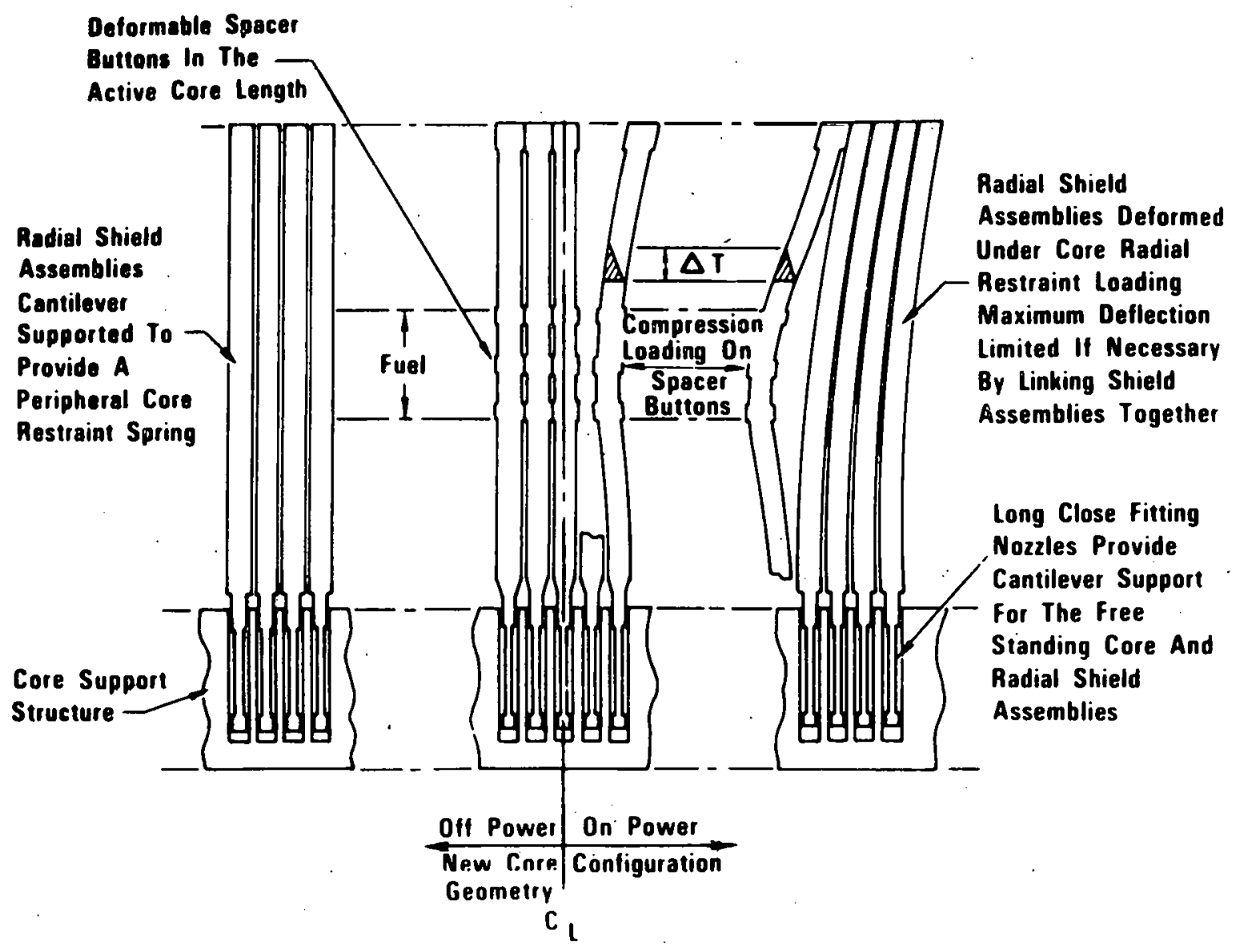

Fig. 4. Limited free bow core restraint concept (from Ref. 7) 


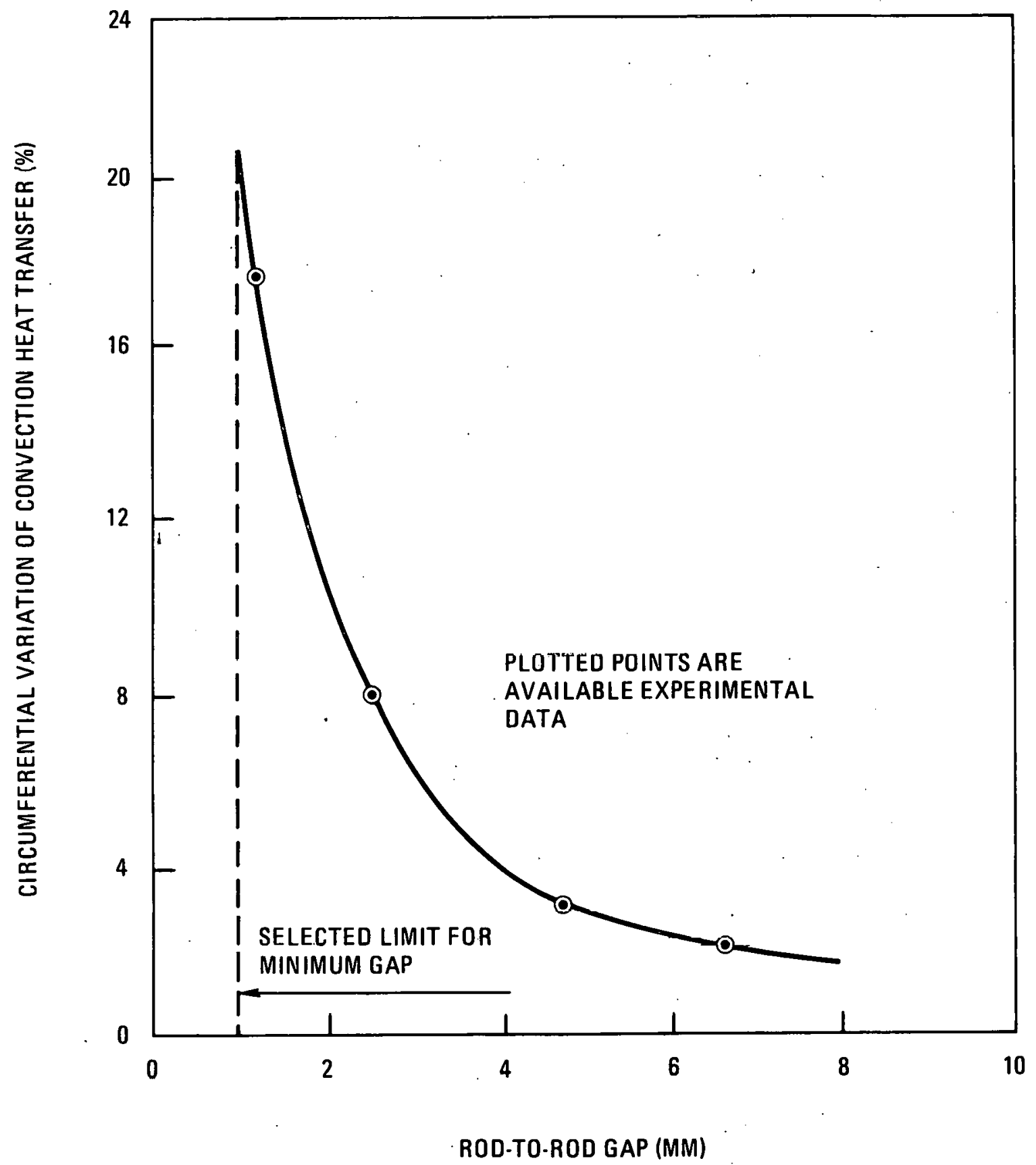

Fig. 5. Basis for mintmum rod gap spacing 